\title{
DEMOGRAPHICS OF NUCLEAR ACTIVITY IN NEARBY GALAXIES
}

LUIS C. HO

Harvard-Smithsonian Center for Astrophysics

ALEXEI V. FILIPPENKO

Department of Astronomy, University of California, Berkeley

AND

WALLACE L. W. SARGENT

Palomar Observatory, California Institute of Technology

\section{The Palomar Survey of Nearby Galaxies}

Between 1984 and 1990 we conducted a survey at Palomar Observatory (Filippenko and Sargent 1985) to quantify the luminosity function of nearby active galactic nuclei (AGNs). The Hale $5 \mathrm{~m}$ telescope was used to obtain high-quality, moderate-resolution optical spectra of a nearly statistically complete sample of about 500 bright $\left(B_{T} \leq 12.5 \mathrm{mag}\right)$, northern $\left(\delta>0^{\circ}\right)$ galaxies (see Ho et al. 1995 for details). The survey has now been completed, and the first scientific results are reported in a series of papers by Ho et al. (1997a, b, c).

\section{Highlights}

Some of the key findings are as follows.

1. AGNs are very common in nearby galaxies (Fig. 1). At least $40 \%$ of all galaxies brighter than $B_{T}=12.5 \mathrm{mag}$ show spectral signatures of nuclear "activity" of probably nonstellar nature. The emission-line nuclei are classified as Seyferts, LINERs, or LINER/H II-region composites, and most have very low luminosities in comparison with traditionally studied AGNs. The luminosities of the $\mathrm{H} \alpha$ emission line range from $10^{37}$ to $10^{41}$ ergs $\mathrm{s}^{-1}$, with a median value of $\sim 10^{39} \mathrm{ergs} \mathrm{s}^{-1}$. 

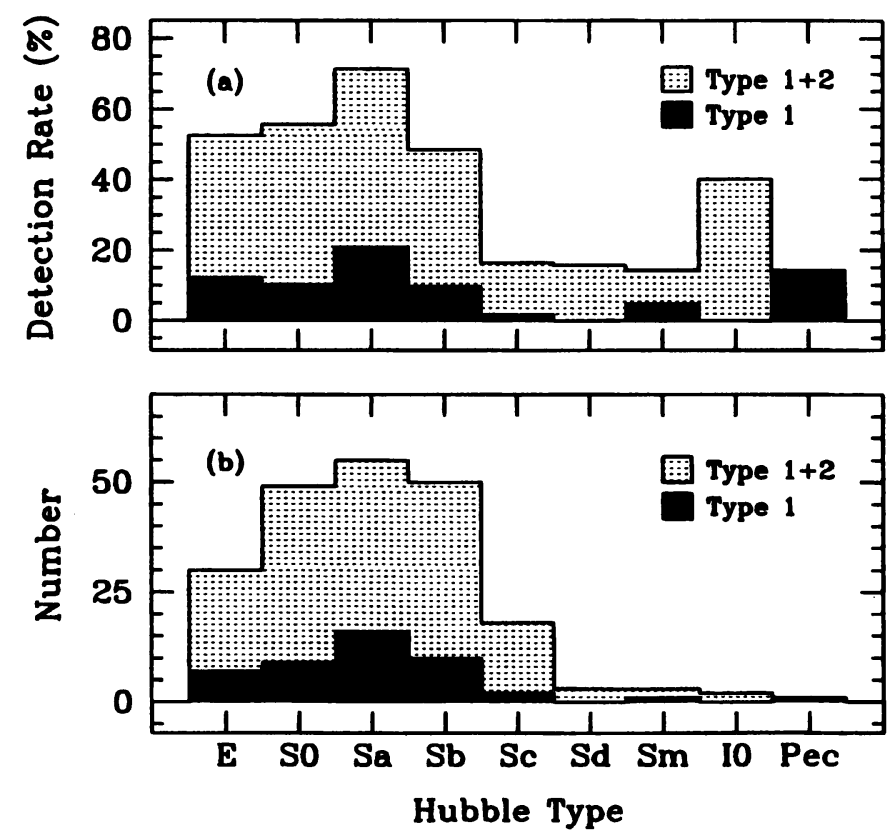

Figure 1. (a) Detection rate and (b) number distribution of AGNs as a function of Hubble type in the Palomar survey. "Type 1" AGNs (those with broad H $\alpha$ ) are shown separately from the total population (types 1 and 2 ).

2. The detectability of AGNs depends strongly on the morphological type of the galaxy, being most common in early-type systems (E-Sbc).

3. LINERs make up the bulk (2/3) of the AGN population and a sizable fraction $(1 / 3)$ of all galaxies.

4. We found a significant number of nuclei showing a faint, broad (FWHM $\approx 1000-4000 \mathrm{~km} \mathrm{~s}^{-1}$ ) $\mathrm{H} \alpha$ emission line that qualitatively resembles emission arising from the conventional broad-line region of "classical" Seyfert 1 nuclei and QSOs. About half of these "type 1" AGNs are formally classified as LINERs. This furnishes strong evidence that at least some LINERs are indeed genuine AGNs.

\section{References}

Filippenko, A. V., and Sargent, W. L. W. 1985, ApJS, 57, 503

Ho, L. C., Filippenko, A. V., and Sargent, W. L. W. 1995, ApJS, 98, 477

Ho, L. C., Filippenko, A. V., and Sargent, W. L. W. 1997a, ApJS, 112, 315

Ho, L. C., Filippenko, A. V., and Sargent, W. L. W. 1997b, ApJ, 487, 568

Ho, L. C., Filippenko, A. V., Sargent, W. L. W., and Peng, C. Y. 1997c, ApJS, 112, 391 